\title{
Morfometria do encéfalo e formação do circuito arterioso em coelhos Nova Zelândia
}

\author{
Janaina de Oliveira Portugal ${ }^{1}$ \\ Sara Otoni da Silva Moraes ${ }^{1}$ \\ Marcelo Salvador Gomes ${ }^{1}$ \\ Rayssa Mariano Reis ${ }^{2}$ \\ Marcelo Abidu-Figueiredo ${ }^{1 *}$ \\ ${ }^{1}$ Universidade Federal Rural do Rio de Janeiro \\ BR-465, km 7, Campus Seropédica, CEP 23890-00, Seropédica - RJ, Brasil \\ ${ }^{2}$ Universidade Federal Fluminense, Niteroi - RJ, Brasil \\ * Autor para correspondência \\ marceloabidu@gmail.com
}

Submetido em 03/06/2013

Aceito para publicação em 08/01/2014

\section{Resumo}

O objetivo deste artigo é descrever as principais ramificações da artéria basilar, a formação do circuito arterioso, e a morfometria do encéfalo em coelhos adultos de ambos os sexos. Foi realizada canulação da aorta torácica através de incisão lateral, fixação com solução de formaldeído a $10 \%$ e preenchimento do sistema arterial com solução de látex corado. Procedeu-se à craniotomia, remoção e morfometria do encéfalo, além de dissecção para a observação das ramificações principais da artéria basilar e da formação do circuito arterioso. A média e erro padrão do comprimento da artéria basilar foram de $1,293 \mathrm{~cm} \pm 0,024$ nas fêmeas e $1,227 \mathrm{~cm}$ $\pm 0,025$ nos machos. Em todos os coelhos dissecados, a artéria basilar se originou da anastomose das artérias vertebrais, direita e esquerda, emitindo a artéria cerebelar caudal, ramos variados para bulbo e ponte, ramos terminais e artérias cerebelares rostrais. As artérias da base do encéfalo estiveram na dependência dos sistemas carótico e vértebro-basilar. O circuito arterioso do cérebro estava fechado rostralmente em 10 machos e 12 fêmeas e fechado caudalmente em todos os animais dissecados.

Palavras-chave: Cérebro; Coelho; Vascularização arterial

\section{Abstract}

Brain morphometry and arterial circuit formation in New Zealand rabbits. This article aims to describe the main ramifications of basilar artery, arterial circuit formation, and brain morphometry in adult rabbits of both sexes. We performed cannulation of the thoracic aorta through lateral incision, fixation with $10 \%$ formaldehyde solution, and filling of the arterial system with colored latex solution. We performed craniotomy, brain removal and morphometry, as well as dissection for observing the main ramifications of the basilar artery and arterial circuit formation. Mean and standard error of the basilar artery length were $1.293 \mathrm{~cm} \pm 0.024$ in females and $1.227 \mathrm{~cm} \pm 0.025$ in males. In all rabbits dissected, the basilar artery stemmed from the anastomosis of vertebral arteries, right and left, giving rise to the caudal cerebellar artery, varied branches to bulb and pons, 
terminal branches, and rostral cerebellar arteries. Arteries at the base of the brain depended on the carotid and vertebral-basilar systems. The arterial brain circuit was rostrally closed in 10 males and 12 females and caudally closed in all animals dissected.

Key words: Arterial vascularization; Brain; Rabbit

\section{Introdução}

Nos últimos anos tem se observado grande avanço nas técnicas de diagnóstico por imagem, que se destinam à investigação das estruturas do sistema nervoso central (QUIÑONES-HINOJOSA et al., 2006). Acompanhando o mesmo crescimento, muitas doenças degenerativas também passaram a ser diagnosticadas com mais frequência e precisão. Tais patologias merecem estudos mais pormenorizados, entretanto encontram-se dificuldades quanto à utilização de modelos, visto que a legislação vigente não permite pesquisas com seres humanos.

O uso de animais como modelo experimental acontece há muito tempo e tem possibilitado muitos avanços na pesquisa biomédica, pois permitem controlar numerosas variáveis que não podem ser obtidas em estudos com seres humanos.

Dentre os modelos experimentais mais usados podemos destacar o suíno em patologias do sistema nervoso e urogenital (PEREIRA-SAMPAIO et al., 2004), carnívoros em patologias osteoarticulares e condições de septicemia, roedores e coelhos com testes de novas drogas e patologias relacionadas ao sistema urogenital (MAIA et al., 2006). O coelho também tem sido bastante utilizado como modelo experimental em cranioplastia, bem como treinamento em cirurgias estereotáxicas destinadas a ressecções, punções e biópsias para diagnóstico em tumores de diferentes linhagens celulares, que precisam de abordagem terapêutica diferenciada (ZAGZAG et al., 1998).

Apesar disto, valores morfométricos de referência e vascularização do encéfalo de coelhos ainda são escassos na literatura, necessitando de maior investigação. O objetivo deste trabalho é fornecer parâmetros morfométricos e vasculares do encéfalo de coelhos, subsidiando a pesquisa aplicada em trabalhos correlatos.

\section{Material e Métodos}

Os estudos de dissecção anatômica foram realizados em 44 coelhos, 21 machos e 23 fêmeas, pesando em media $2,5 \mathrm{~kg}$. Todos os animais utilizados faziam parte das aulas práticas das disciplinas de Cirurgia e foram cedidos à área de Anatomia Animal. Os animais já haviam sido submetidos à eutanásia, com administração de superdosagem de tiopental sódico. Foram previamente tricotomizados, identificados e posicionados em decúbito lateral direito. Em seguida, o tórax foi aberto e dissecado para evidenciação da aorta torácica, que foi canulada. Desse modo, o sistema arterial foi "lavado" com solução fisiológica de $\mathrm{NaCl}$ a $0,9 \%$, sendo realizado a seguir a fixação com solução de formaldeído a $10 \%$, conforme técnica anatômica padrão. Em seguida, injetou-se pela cânula solução aquosa (diluição 1:1) de Petrolátex S-65 (Refinaria Duque de Caxias (REDUC) da Petrobrás, Duque de Caxias-RJ) misturado ao corante (Suvinil xadre $^{\circledR}$ ). Os animais permaneceram acondicionados em cubas com solução de formaldeído a $10 \%$ por cinco dias para polimerização do látex. Os encéfalos foram removidos do crânio para serem submetidos à morfometria. Todas as mensurações foram realizadas pelo mesmo observador, utilizando um paquímetro digital (ZAAS Precision, Amatools ${ }^{\circledR}$ ).

O comprimento dos hemisférios cerebrais foi obtido através da distância entre os pólos frontal e occipital de cada hemisfério, a largura entre a distância do sulco mediano dorsal até o pólo temporal respectivo e a altura compreendida entre a base do lobo piriforme e a superfície dorsal do respectivo hemisfério. Também foram feitas medidas do comprimento e largura do cerebelo.

Os vasos da base do encéfalo foram dissecados e fotografados para documentação e denominados de acordo com a Nomenclatura Anatômica Veterinária (SCHALLER, 1999). Também foram feitos desenhos esquemáticos. Esta publicação faz parte do projeto 
intitulado Anatomia Topográfica e Sistêmica do Coelho: estudo destinado a pratica cirúrgica, radiológica e experimental, que já foi submetido e aprovado pelo comitê de ética e pesquisa da Universidade Federal Rural do Rio de Janeiro, de número 23083.002379/2007-08.

\section{Análise estatística}

Foram obtidas a média e o erro padrão da média das medidas do encéfalo e da artéria basilar em ambos os sexos e comparadas através do Teste t não pareado, considerando $\mathrm{p} \leq 0,05$ significativo.

\section{Resultados}

Os resultados da morfometria do encéfalo e da artéria basilar em coelhos do sexo masculino e feminino estão representados na Tabela 1.

\section{Artéria basilar}

A média mais erro padrão do comprimento da artéria basilar foi de 1,227 $\pm 0,025$ nos machos e 1,293 $\pm 0,024$ $(\mathrm{p}=0,069)$ nas fêmeas. Tanto nos machos quanto nas fêmeas, as artérias vertebral direita e esquerda, foram as responsáveis pela formação da artéria basilar. Apresentouse única em 23 (100\%) fêmeas, e em 20 (95\%) machos; e em apenas um (5\%) macho se apresentou dupla. Em ambos os sexos, a artéria basilar seguiu de forma retilínea em direção à base do encéfalo (Figura 1).
FIGURA 1: Fotomacrografia da vista ventral do encéfalo mostrando as ramificaçoes principais da arteria basilar. av : artéria vertebral ab: artéria basilar acr: artéria cerebelar rostral.

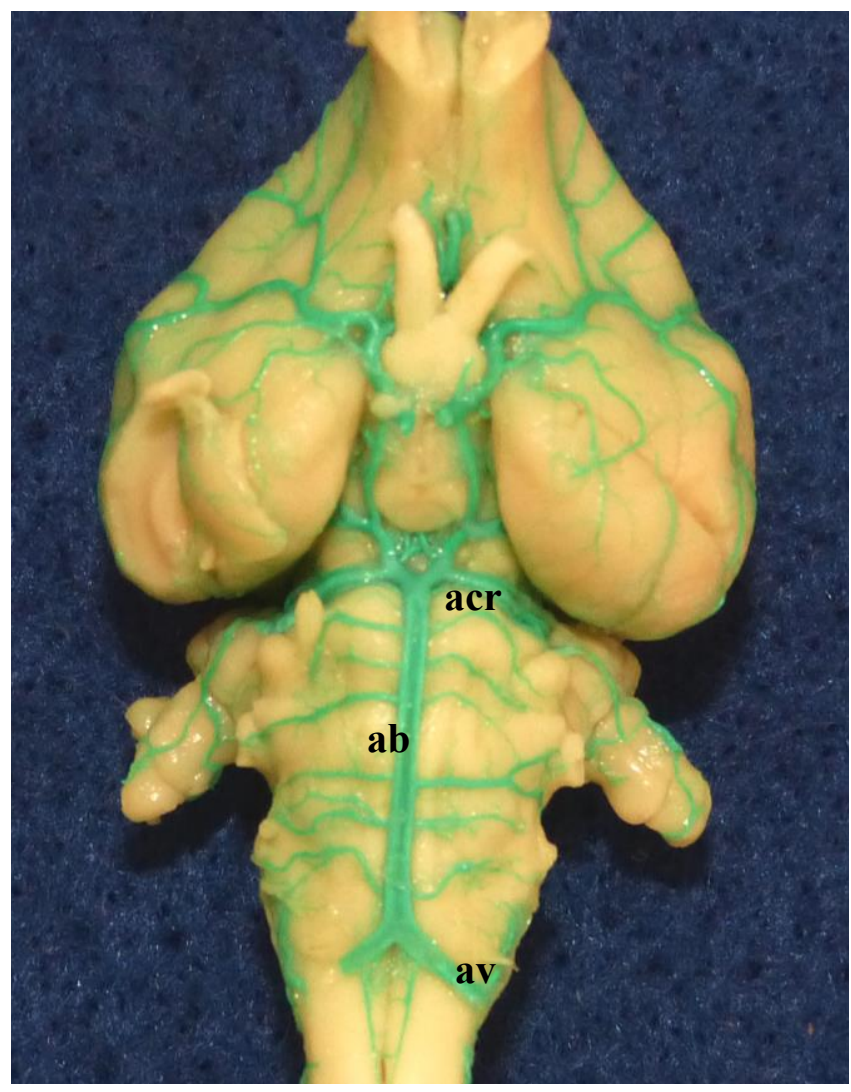

A artéria basilar emitiu como primeiro ramo a artéria cerebelar caudal única em ambos os antímeros e em ambos os sexos. Este vaso apresentou simetria de posição em $17(80,90 \%)$ machos e 17 (73, 90\%) fêmeas

TABELA 1: Média e erro padrão da média das medidas em centímetros do encéfalo e da artéria basilar em coelhos de ambos os sexos.

\begin{tabular}{lcc}
\hline & \multicolumn{2}{c}{ Coelhos } \\
\cline { 2 - 3 } & Machos & Fêmeas \\
\hline Comprimento do Hemisfério Direito & $2,655 \pm 0,034$ & $2,520 \pm 0,077(\mathrm{p}=0,1254)$ \\
\hline Comprimento do Hemisfério Esquerdo & $2,801 \pm 0,144$ & $2,600 \pm 0,032(\mathrm{p}=0,1718)$ \\
\hline Altura do Hemisfério Direito & $1,430 \pm 0,016$ & $1,392 \pm 0,013(\mathrm{p}=0,0795)$ \\
\hline Altura do Hemisfério Esquerdo & $1,432 \pm 0,022$ & $1,448 \pm 0,013(\mathrm{p}=0,6043)$ \\
\hline Largura do Hemisfério Direito & $1,196 \pm 0,018$ & $1,121 \pm 0,015(\mathrm{p}=0,037)$ \\
\hline Largura do Hemisfério Esquerdo & $1,171 \pm 0,014$ & $1,121 \pm 0,020(\mathrm{p}=0,0582)$ \\
\hline Comprimento do Cerebelo & $1,256 \pm 0,034$ & $1,225 \pm 0,034(\mathrm{p}=0,528)$ \\
\hline Largura do Cerebelo & $1,550 \pm 0,029$ & $1,538 \pm 0,023(\mathrm{p}=0,754)$ \\
\hline Comprimento da a basilar & $1,227 \pm 0,025$ & $1,293 \pm 0,024(\mathrm{p}=0,069)$ \\
\hline
\end{tabular}


e consequentemente assimetria em quatro $(19,10 \%)$ machos e seis $(26,10 \%)$ fêmeas. Em seguida a artéria basilar emitiu ramos variáveis para bulbo e ponte em ambos os antímeros em machos e fêmeas. Nos machos a média, mais erro padrão da média dos ramos para o antímero direito foi de 5,429 $\pm 0,2723$ e para o esquerdo de $5,190 \pm 0,2140(p=0,4958)$. Nas fêmeas a média, mais erro padrão da média dos ramos para o antímero direito foi de 5,522 $\pm 0,2424$ e para o esquerdo de 5,609 $\pm 0,1859(\mathrm{p}=0,7772)$. No ponto de bifurcação, a artéria basilar em sua porção rostral emitiu ramos para ambos os antímeros denominados de ramos terminais da artéria basilar. A artéria cerebelar rostral se apresentou de forma simétrica em 19 (90,47\%) machos e 21 (91,30\%) fêmeas.

A assimetria foi observada em dois (9,53\%) machos e duas $(8,7 \%)$ fêmeas. Em relação ao número, a artéria cerebelar rostral se apresentou única em ambos os antímeros em 13(61,90\%) machos e 12 (52,17\%) fêmeas, dupla em ambos os antímeros em um (4,76\%) macho e quatro $(17,39 \%)$ fêmeas, dupla no antímero esquerdo e única no direito em quatro $(19,4 \%)$ machos apenas, dupla no antímero direito e única no esquerdo em três $(14,28 \%)$ machos e sete $(30,43 \%)$ fêmeas. A artéria cerebelar rostral se originou do ramo terminal da artéria basilar em 17 (80,95\%) machos e 21 (91,30\%) fêmeas e da artéria basilar, na altura da ponte em quatro $(19,04 \%)$ machos e uma ( $4,34 \%$ ) fêmea, todos de forma simétrica em ambos os antímeros. Em apenas uma (4,34\%) fêmea, a artéria cerebelar rostral esquerda se originou da artéria basilar na altura da ponte enquanto que a correspondente direita se originou do ramo terminal da artéria basilar.

\section{Formação do circuito arterioso do cérebro}

O circuito arterioso cerebral do coelho formou uma figura geométrica irregular, envolvendo rostralmente a base dos nervos ópticos, o túber cinéreo e caudalmente o corpo mamilar e fossa interpeduncular. Estende-se desde a fissura longitudinal do cérebro na altura da base do trígono olfatório até o sulco que separa a ponte dos pedúnculos cerebrais.

Os vasos que o delimitavam foram: rostralmente, ramo rostral da artéria carótida interna, artérias cerebrais rostrais e artéria comunicante rostral; e caudalmente, pelos ramos caudais da artéria carótida interna (artérias comunicantes caudais) e os ramos terminais da artéria basilar (Figura 2).

FIGURA 2: Fotomacrografia da vista ventral do encéfalo mostrando a formação do circuito arterioso. rcac : ramo caudal da artéria carótida rrac: ramo rostral da artéria carótida acm: artéria cerebral média acr: artéria cerebral rostral

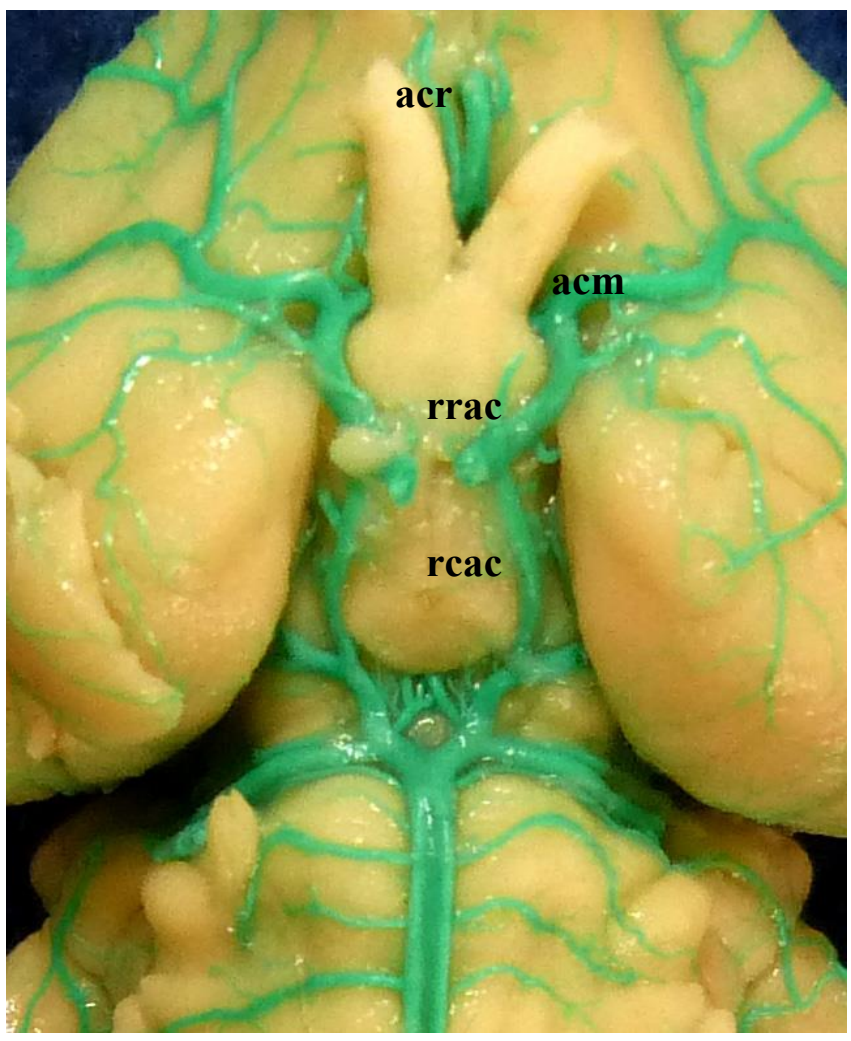

O circuito arterioso do cérebro apresentouse fechado rostralmente pela presença da artéria comunicante rostral em 10 (47,62\%) machos e 12 $(52,17 \%)$ fêmeas. Caudalmente esteve fechado em todos os animais dissecados.

A artéria cerebral caudal apresentou simetria de posição em todos os coelhos examinados (100\%). Apresentou-se única em ambos os antímeros em 18 $(85,71 \%)$ machos e $20(86,95 \%)$ fêmeas, dupla no antímeros esquerdo e única no direito em dois machos $(9,52 \%)$ e uma $(4,35 \%)$ fêmea, dupla no antímero direito e única no esquerdo em um $(4,76 \%)$ macho e duas $(4,35 \%)$ fêmeas. A artéria cerebral caudal teve origem no ponto de união entre o ramo terminal da artéria basilar 
com ramo caudal da artéria carótida em 100\% dos coelhos. A artéria cerebral média apresentou simetria de posição em $16(79,19 \%)$ machos e $20(86,95 \%)$ fêmeas, assimetria em cinco $(28,80 \%)$ machos e três $(13,04 \%)$ fêmeas. Apresentou-se dupla em ambos os antímeros em $100 \%$ dos coelhos; e se originou do ramo rostral da artéria carótida interna em todos os animais dissecados. A artéria cerebral rostral apresentou-se de forma retilínea e fusionada e se originou diretamente do ramo rostral da artéria carótida interna em todos os animais.

\section{Discussão}

Morfometria é o estudo científico das dimensões de estruturas anatômicas macroscópicas dos indivíduos, com especial referência às suas medidas lineares e pesos. Estas medidas são muitas vezes feitas por medições diretas em amostras fixadas em solução de formaldeído, especialmente no cérebro. Os resultados variam em precisão e ainda são motivos de discussão (PETERS et al., 1998).

Olapade et al. (2005) realizaram morfometria do encéfalo de ovinos separando-os por sexo, peso e idade; e concluíram que não houve diferença nos parâmetros do encéfalo e cérebro em relação ao sexo e a idade; entretanto animais mais pesados apresentaram aumento no comprimento do cérebro e do encéfalo. $\mathrm{Na}$ presente investigação com exceção da largura do hemisfério cerebral, não houve diferença nas medidas do cérebro e cerebelo entre os sexos. Ribeiro et al. (2005) realizaram morfometria em 81 hemisférios cerebrais humanos comparando os dois sexos e concluíram que os homens apresentaram maior comprimento e maior peso encefálico, diferente dos resultados obtidos na presente investigação onde só houve diferença da largura dos hemisférios. Igado e Aina (2010), em estudo referente às medidas do cérebro de codorna (Coturnix japonica) usando machos e fêmeas, observaram que as fêmeas apresentaram média dos valores de comprimento, peso, e profundidade do cérebro, superiores aos machos, diferente dos resultados obtidos na presente pesquisa onde os machos apresentaram maior largura dos hemisférios.

\section{Morfometria e origem da artéria basilar}

Não houve diferença na medida do comprimento da artéria basilar entre o sexo dos coelhos estudados $(\mathrm{p}=0,069)$. Na literatura consultada não foram observados valores correspondentes à medida desse vaso.

A artéria basilar, tanto nos machos quanto nas fêmeas, formou-se a partir da anastomose das artérias vertebrais, direita e esquerda semelhante ao observado no gato (LIMA et al., 2006; GOMES et al., 2012a). Resultados diferentes foram obtidos por Jewell (1952) em cães, onde a artéria vertebral se juntava à artéria espinhal ventral na superfície ventral da medula espinhal, após receber anastomose das artérias occipitais originando rostralmente a artéria basilar. De La Torre et al. (1962), entretanto, relataram em cães que esta artéria era formada pela união dos ramos dorsais direito e esquerdo do ramo occipito-vertebral, semelhante aos resultados obtidos por Gillilan (1976), que também usou o cão e o gato. Diferente dos autores acima, Pais et al. (2009) observaram que, em todos os cães analisados, as artérias vertebrais direita e esquerda foram as responsáveis pela formação da artéria basilar, corroborando com os resultados da presente investigação.

Em estudo com fetos de bovinos azebuados (MELO; PRADA, 1998) e com fetos de búfalos sem raça definida (FARIA; PRADA, 2001), a artéria basilar se apresentou como um vaso ímpar resultante da convergência dos ramos caudais, direito e esquerdo, da artéria carótida; diferente do observado por Ocal et al. (1999), em camelo (Camelus dromedrarius) e Brudnicki (2000), em caprinos, onde em ambos os casos a artéria basilar era formada pela anastomose das artérias vertebrais e artéria espinhal ventral. Os resultados acima diferem dos obtidos na presente investigação, onde a artéria basilar se formou exclusivamente a partir da união das artérias vertebral direita e esquerda.

Almeida e Campos (2011) observaram que no jacaré do papo amarelo (Caiman latirostris) a artéria basilar era formada a partir da anastomose das partes convergentes do ramo caudal da artéria carótida interna, arranjo semelhante ao observado por Nazer e Campos (2011), no cérebro de avestruz, onde a artéria basilar apresentou-se como um vaso único em $80 \%$, de forma 
dupla em $13,3 \%$ e em forma de ilha em $6,7 \%$ das amostras, diferente dos resultados da presente pesquisa no que se refere à formação da artéria basilar; entretanto se assemelha aos resultados dos autores supra citados, pois em coelhos também houve predomínio da forma única.

Variações na artéria basilar foram descritas por Paiva-de-Souza et al. (2010), em equinos mestiços com a frequência variável da artéria caroticobasilar, uma anastomose entre a artéria carótida interna e a artéria basilar observada na face ventral do tronco encefálico. Depedrine e Campos (2003) observaram que na raposa (Pseudalopex gymnocercus), a artéria basilar originou-se da artéria vertebral direita e esquerda e pela bifurcação terminal rostral da artéria espinhal ventral, entretanto, de acordo com Wiland (1966), a artéria basilar na raposa forma-se através da união dos ramos da artéria vertebral e occipital. Em ambos os autores acima citados, os resultados obtidos diferem dos verificados na presente investigação. Em estudo realizado com roedores da espécie Hystrix cristata e Spalax leucodon, Aydin et al. (2005; 2008), respectivamente, observaram que em ambas as espécies a artéria basilar originouse da fusão das artérias vertebral direita e esquerda; este mesmo arranjo foi observado por Reckziegel et al. (2001), em estudo realizado em capivaras (Hydrochoerus hydrochoeris) e também nos resultados obtidos na presente investigação.

Em equinos Puro Sangue Inglês, Campos et al. (2003) observaram que a artéria basilar formou-se a partir da convergência das artérias occipitais direita e esquerda, diferente do que foi observado na presente pesquisa.

Lima et al. (2005), em estudo realizado com encéfalos de suínos da linhagem Camborough 22, Ferreira e Prada (2005), em suínos sem raça definida, e Oliveira e Campos (2005), no javali (Sus scrofa scrofa), observaram que a rede admirável epidural caudal e ainda a artéria espinhal ventral foram as responsáveis pela formação da artéria basilar, diferente dos resultados obtidos na presente investigação.

Em chinchilas (Chinchilla lanígera), Araújo e Campos (2005) observaram que em 96,7\% a artéria basilar formou-se pela convergência das artérias vertebral direita e esquerda, enquanto que, em 3,3\%, foi formada pela artéria carótida interna esquerda. Os resultados desses autores, apesar de não estarem expressos separados pelo sexo, assemelham-se, em parte, com os do presente estudo, pois em $100 \%$ dos animais dissecados as artérias vertebrais, direita e esquerda, foram responsáveis pela formação da artéria basilar.

Azambuja (2006) observou que no ratão do banhado (Myocastor ciypus) a artéria basilar formava-se a partir da anastomose dos ramos terminais das artérias vertebral direita e esquerda, semelhante ao que fora observado em coelhos na presente investigação, e aos resultados de Ferreira e Prada (2001), em estudo sobre os vasos da base do encéfalo do macaco-prego (Cebus apella), Barreiro et al. (2012), em estudo realizado em quatis, e Lima et al. (2013), em tamanduás-mirim.

\section{Ramificações principais}

Em todos os animais dissecados, a artéria basilar emitiu como ramos principais; a artéria cerebelar caudal que se apresentou de forma simétrica e assimétrica em relação ao antímero, seguida de ramos variados em número, e de menor calibre para ponte e bulbo e como último ramo, as artérias cerebelares rostrais, direita e esquerda. Esses resultados assemelham-se aos obtidos por Lima et al. (2006) e Gomes et al. (2012a), para gatos, e Alcântara e Prada (1996a; 1996b), para o cão. Entretanto, Barreiro et al. (2012) e Lima et al. (2013) observaram a presença da artéria cerebelar média, vaso este que não foi observado em coelhos.

\section{Formação do circuito arterioso do cérebro}

O circuito arterioso do cérebro em animais domésticos e selvagens pode ser usado para criar modelos que simulem o homem, com o propósito de conduzir diferentes experimentos. Sendo assim o estudo morfológico comparativo detalhado desses vasos tornase essencial e relevante.

Estudos do circuito arterioso da base do encéfalo de diferentes espécies demonstram muitas variações. 
Nazer e Campos (2011), em avestruz (Struthio camelus) observaram que este se apresentou sempre aberto rostralmente e em $80 \%$ dos casos aberto caudalmente. Almeida e Campos (2011), no jacaré (Caiman latirostris) verificaram que este foi dependente exclusivamente do sistema carótico, e se apresentou fechado rostralmente e caudalmente em todos os espécimes estudados, semelhante aos resultados obtidos por Azambuja (2006), em estudo com o Ratão do Banhado (Myocastor coypus) embora o circuito nesses animais fosse suprido exclusivamente pelo sistema vértebro-basilar. $\mathrm{Na}$ presente investigação o circuito se mostrou fechado rostralmente pela presença da artéria comunicante rostral em 10 machos e 12 fêmeas, fechado caudalmente em $100 \%$ e na dependência exclusiva do sistema carótico e vértebro-basilar, semelhante ao observado no gato (LIMA et al., 2006; GOMES et al., 2012b).

O circuito arterioso do suíno (Sus scrofa domesticus) é fechado rostralmente pela presença constante da artéria comunicante rostral em $100 \%$ dos casos e caudalmente pela presença dos ramos terminais direito e esquerdo da artéria basilar (FERREIRA; PRADA, 2005) semelhante ao observado no Javali (Sus scrofa scrofa) (OLIVEIRA; CAMPOS,2005) e diferentemente dos observados na presente investigação.

No gato (LIMA et al., 2006; GOMES et al., 2012b) observaram que o circuito se mostrou fechado rostralmente pela presença da artéria comunicante rostral em $60 \%$ dos casos e caudalmente fechado pela presença dos ramos caudais das artérias carótidas do encéfalo e ramos terminais da artéria basilar em todas as amostras semelhante ao observado na presente pesquisa, onde em $50 \%$ dos coelhos foi observada a artéria comunicante rostral.

Ocal et al. (1999), em camelo (Camelus dromedarius), concluíram que o circuito arterioso teve seu suprimento sanguíneo oriundo da artéria carótida, em cada antímero, da artéria basilar além da artéria comunicante caudal que se originou da rede admirável epidural rostral. Em coelhos deste estudo apesar das semelhanças quanto à formação do circuito (a. carótida e a. basilar) não se observa a formação da artéria comunicante caudal mostrada em camelos.
Araújo e Campos (2005), em chinchilla (Chinchilla lanígera), relataram que a irrigação sanguínea do encéfalo é formada pelo sistema vértebro-basilar, porém em pequenas proporções, pela artéria carótida interna esquerda ou pelo sistema-vértebro basilar com contribuição da artéria carótida interna esquerda. Reckziegel et al. (2001), em capivara (Hydrochoerus hydrochaeris), verificaram que é depende unicamente do sistema vértebro-basilar corroborando parcialmente com os resultados observados em coelhos deste estudo, onde além da participação do sistema vértebro-basilar pode-se observar o sistema carótico. No circuito arterioso do esquilo vermelho (Sciurus vulgaris) a artéria carótida interna não contribuiu para o suprimento sanguíneo do circuito arterioso; este é feito pela artéria basilar (AYDIN et al., 2008). Arranjo morfológico semelhante foi observado por Aydin et al. (2009), no esquilo (Spermophilus citellus) e por Aydin et al. (2005), no porco-espinho (Hystrix cristata), diferentemente dos nossos resultados onde além da artéria basilar há participação da artéria carótida. Lima et al. (2006), Gomes et al. (2012b), em gatos, mostraram que o circuito arterioso estende-se desde a ponte até rostralmente ao quiasma óptico, sendo representado pela artéria carótida do encéfalo direita e esquerda, através de seus correspondentes ramos rostrais e caudais e, ainda pelos ramos terminais em ambos os antímeros da artéria basilar em todas as preparações, corroborando com os resultados da presente investigação.

Em relação à forma do circuito arterioso em gatos, Lima et al. (2006) relataram que o arranjo dos ramos rostrais da carótida lembram uma figura elipsoide disposta transversalmente em relação à base do encéfalo, diferentemente, Gomes et al. (2012b), também em gatos, observaram que o circuito arterioso não apresentou nenhuma configuração semelhante a uma forma geométrica. Entretanto, observaram predomínio da forma retilínea nos machos e cruzada nas fêmeas em relação à artéria cerebral rostral. $\mathrm{Na}$ presente investigação o arranjo formado pelos ramos rostrais da carótida não formaram nenhum forma geométrica, alem disso artéria cerebral rostral se apresentou retilínea e fusionada em todos os coelhos (100\%). Assim concluiuse que; o circuito arterioso do coelho esta na dependência do sistema carótico e vértebro-basilar, que existe 
um predomínio da forma retilínea da artéria cerebral rostral, e que a presença da artéria comunicante rostral independe do sexo do animal.

\section{Referências}

ALCÂNTARA, M. A.; PRADA, I. L. S. Arteries of basis of encephalon in dogs (Canis familiaris, Linnaeus, 1758). I. Anatomical study of sources and behavior. Brazilian Journal of Veterinary Research and Animal Science, São Paulo, v. 33, n. 2, p. 67-71, 1996a.

ALCÂNTARA, M. A.; PRADA, I. L. S. Arteries of basis of encephalon in dogs (Canis familiaris, Linnaeus, 1758). II. Formation and behavior of the encephalon arterial circuit. Brazilian Journal of Veterinary Research and Animal Science, São Paulo, v. 33, n. 2, p. 72-76, 1996 b.

ALMEIDA, L.; CAMPOS, R. A. Systematic study of the brain base arteries in broad-snouted caiman (Caiman latirostris). Journal of Morphological Science, São Paulo, v. 28, n. 1, p. 62-68, 2011.

ARAÚJO, A. P. C.; CAMPOS, R. A. Systematic study of the brain base arteries and their blood supply sources in the chinchilla (Chinchilla lanigera, Molina, 1782). Brazilian Journal of Morphological Science, São Paulo, v. 22, n. 4, p. 221-232, 2005.

AYDIN, A.; DINC, G.; KARAN, M.; OZDEMIR, D.; YILMAZ, $\mathrm{S}$. The morphology of circulus arteriosus cerebri in the porcupine (Hystrix cristata). Veterinarni Medicina, Prague, v. 50, n. 3, p. 131-135, 2005.

AYDIN, A.; ILGÜN, R.; OZKAN, Z. E.; YILMAZ, S. Morphological investigations on the circulus arteriosus cerebri in mole-rats (Spalax leucodon). Anatomia Histologia Embryologia, Berlin, v. 37, n. 3, p. 219-222, 2008.

AYDIN, A.; ILGUN, S.; OZKAN, Z. E.; YILMAZ, S. Morphology of the circulus arteriosus cerebri in the ground squirrel (Spermophilus citellus). Veterinarni Medicina, Prague, v. 54, n. 11, p. 537-542, 2009.

AZAMBUJA, R. C. Sistematização das artérias da base do encéfalo e suas fontes de suprimento sanguíneo em nutria (Myocastor coypus). 2006. 150 f. Dissertação (Mestrado em Ciências Veterinárias) - Universidade Federal do Rio Grande do Sul, Porto Alegre. 2006.

BARREIRO, J. R.; CARVALHO, A. F.; FRANCIOLLI, A. L. R.; FERREIRA, G. J. B. C.; FERREIRA, J. R.; AMBRÓSIO, C. E.; MIGLINO, M. A. Morfologia dos vasos da base do encéfalo do quati (Nasua nasua). Pesquisa Veterinária Brasileira, Rio de Janeiro, v. 32, n. 6, p. 567-572, 2012.

BRUDNICKI, W. Basilar arteries of the brain in domestic goat (Capra hircus). Electronic Journal of Polish Agricultural Universities, Cracow, v. 3, n. 1, p. 1-4, 2000.

CAMPOS, A.; DOS SANTOS, D.; DOS SANTOS JUNIOR, I.; PRADA, I. S. Artérias da base do encéfalo de eqüinos. Sistema occipito-basilar. Brazilian Journal of Veterinary Research and Animal Science, São Paulo, v. 40, n. 1 p. 107- 117, 2003.

DE LA TORRE, E.; MICHELL, O. C.; NETSKY, M. A. Anatomic and angiographic studies of de vertebral basilar system in dog.
American Journal of Anatomy, Philadelphia, v. 110, n. 2, p. $187-$ 198, 1962.

DEPEDRINI, J. S.; CAMPOS, R. A systematic study of the brain base arteries in the pampas fox (Dusicyon gymnocercus). Brazilian Journal of Morphological Science, São Paulo, v. 20, n. 3, p. 181188, 2003.

FARIA, M. M. M. D.; PRADA, I. L. S. Anatomical behavior of the basilar artery of buffalo fetuses (Bubalus bubalis, Linnaeus, 1758). Revista Brasileira de Saúde e Produção Animal, Salvador, v. 1, n. 2, p. 54-60, 2001.

FERREIRA, C. G.; PRADA, I. L. S. O circuito arterial da base do encéfalo em suínos (Sus scrofa domesticus, Linnaeus, 1758), formação e comportamento. Brazilian Journal of Veterinary Research and Animal Science, São Paulo, v. 42, n. 1, p. 53-60, 2005.

FERREIRA, J. R.; PRADA, I. L. S. Nomenclatura proposta para denominar as artérias da base do encéfalo do macaco-prego (Cebus apella, Linnaeus, 1766). Acta Scientiarum, Maringá, v. 23, n. 2, p. $635-643,2001$.

GILLILAN, L. A. Extra and intra-cranial blood supply to brains of dog and cat. American Journal of Anatomy, Philadelphia, v. 146, n. 3 , p. $237-254,1976$

GOMES, M. S.; DIAS, G. P.; SILVA, M. P. S. S.; SANTOS, C.M .; ABIDU-FIGUEIREDO, M. Circulus arteriosus formation in cat brain. Eurasian Journal of Veterinary Sciences, Selcuk, v. 28, n. 4, p. 204-208, 2012b.

GOMES, M. S.; HERNANDEZ, J. M. F.; ALONSO, L. S.; ABIDUFIGUEIREDO, M. Morfologia e ramos principais da artéria basilar em gatos. Revista Brasileira de Medicina Veterinária, Rio de Janeiro, v. 34, n. 3, p. 206-212, 2012a.

IGADO, O. O.; AINA, O. O. Some aspects of the neurometrics and oculometrics of the Japanese quail (Coturnix coturnix japonica) in Nigéria. Journal of Morphological Science, São Paulo, v. 2 7, n. 3-4, p. 133-135, 2010.

JEWELL, P. A. The anastomoses between internal and external carotid circulation in the dog. Journal of Anatomy, London, v. 86, n. 2, p. 83-94, 1952.

LIMA, E. M. M.; PRADA, I. L. S.; CARNEIRO E SILVA, F. O.; SEVERINO, R. S.; QUAGLIATTO, A. L.; DRUMMOND, S. S.; RODRIGUES, G. S. Estudo anatômico das artérias da base do encéfalo em gatos. Ars Veterinária, São Paulo, v. 22, n. 1, p. 1-7, 2006.

LIMA, E. M. M.; SEVERINO, R. S.; CARNEIRO E SILVA, F. O.; DRUMMOND, S. S.; BOMBONATO, P. P.; CAMPOS, D. B.; RODRIGUES, G. S. Artérias da base do encéfalo do suíno da linhagem camborough 22. Bioscience Journal, Uberlândia, v. 21, n. 2 , p. 137-147, 2005.

LIMA, A. R.; PEREIRA, L. C.; BRANCO E. Anatomia do circuito arterial do encéfalo em tamanduá-mirim. Ciência Rural, Santa Maria, v. 43, n. 2, p. 277-282, 2013.

MAIA, R. S.; ABIDU-FIGUEIREDO, M.; BABINSKI, M. A.; CHAGAS, M. A.; COSTA, W. S.; SAMPAIO, F. J. B. Concentration of elastic system fibers in the corpus cavernosum, corpus spongiosum, and tunica albuginea in the rabbits penis. International Journal of Impotence Research, London, v. 18, n. 2, p. 121-125, 2006.

MELO, A. P. F.; PRADA, I. L. S. Anatomical study of arteries of the base of encephalon in fetuses of zebu crossed bovines. Brazilian 
Journal of Morphological Science, São Paulo, v. 15, n. 2, p. 143 149, 1998.

NAZER, M. B.; CAMPOS, R. Systematization of the brain base arteries in ostrich (Struthio camelus). Journal of Morphological Science, São Paulo, v. 28, n. 4, p. 268-274, 2011.

OCAL, M. K.; ERDEN, H.; OGUT, I.; KARA, M. E. A Quantitative study of the circulus arteriosus cerebri of the camel (Camelus dromedarius).Anatomia Histologia Embryologia, Berlin, v. 28, n. 5-6, p. 271-272, 1999.

OLIVEIRA, J. C. D.; CAMPOS, R. A systematic study of brain base arteries in the wild boar (Sus scrofa scrofa). Anatomia Histologia Embryologia, Berlin, v. 34, n. 4, p. 232-239, 2005.

OLOPADE, J.; OBALOGUN, B. A.; OKE, B. O.; ONWUKA, S. $\mathrm{K}$. Morphometric investigation of the brain of West African dwarf sheep in Nigeria. International Journal of Morphology, Temuco, v. 23, n. 2, p. 99-104, 2005.

PAIS, D.; ARANTES, M.; CASAL, D.; CASIMIRO, M.; O’NEILL, J. G. Brain stem arteries in Canis familiaris - implications in experimental procedures. Brazilian Journal of Morphological Science, São Paulo, v. 26, n. 1, p. 8-11, 2009.

PAIVA-DE-SOUZA, A. V.; XAVIER-SILVA, B.; ANTUNES, M. S.; HERNANDEZ, J. M. F.; SCHERER, P. O.; ABIDUFIGUEIREDO, M. Frequência da artéria caroticobasilar em equinos mestiços: estudo anatômico destinado à pesquisa experimental e ao diagnóstico por imagem. Pesquisa Veterinária Brasileira, Rio de Janeiro, v. 30, n. 8, p. 685-688, 2010.

PEREIRA-SAMPAIO, M. A.; FAVORITO, L. A.; SAMPAIO, F. J. B. Pig kidney: anatomical relationships between the intrarenal arteries and the kidney collecting system. Applied study for urological research and surgical training. Journal of Urology, Baltimore, v. 172, n. 5, p. 2077-2081, 2004.
PETERS, M.; JANCKE, L.; HELANG, Y.; STEINMETZ, H.; SCHLAUS, G.; STAIGER, J. F. Unsolved problems of comparing brain sizes in homosapiens. Brain and Cognition, New York, v. 37, n. 2, p. 254-285, 1998.

QUIÑONES-HINOJOSA, A.; MCDERMOTT, M. W.; SANAI, N.; WARE, M. L. Assessment of image guided accuracy in a skull model: comparison of frameless stereotaxy techniques vs. framebased localization. Journal of Neuro-oncology, Boston, v. 76, n. 1, p. 65-70, 2006.

RECKZIEGEL, S. H.; LINDEMANN, T.; CAMPOS, R. A. Systematic study of the brain base arteries in capybara (Hydrochoerus hydrochaeris). Brazilian Journal of Morphological Science, São Paulo, v. 18, n. 2, p. 104-110, 2001.

RIBEIRO, P. J.; CRICENTI, V. S.; LANCELlOTTI, C. L. P. Encefalometria na face medial do hemisfério cerebral humano: estudo em necropsias. Arquivos de Neuropsiquiatria, São Paulo, v. 63 , n. 2 b, p. $430-436,2005$.

SCHALLER, O. Nomenclatura anatômica veterinária ilustrada. São Paulo: Manole, 1999. 641 p.

WILAND, C. The basilar artery of the brain (A. basilaris cerebri) in foxes. Folia Morphologica, Warszawa, v. 25, n. 4, p. 645-649, 1966.

ZAGZAG, D.; BREM, S.; ROBERT, F. Neovascularization and tumor growth in the rabbit brain. A model for experimental studies of angiogenesis and the blood-brain barrier. American Journal of Pathology, Pennsylvania, v. 131, n. 2, p. 361-372, 1988. 